UCRL-ID-128886

\title{
Efficient Computation of Volume of Hexahedral Cells
}

\author{
J. Grandy
}

October 30, 1997

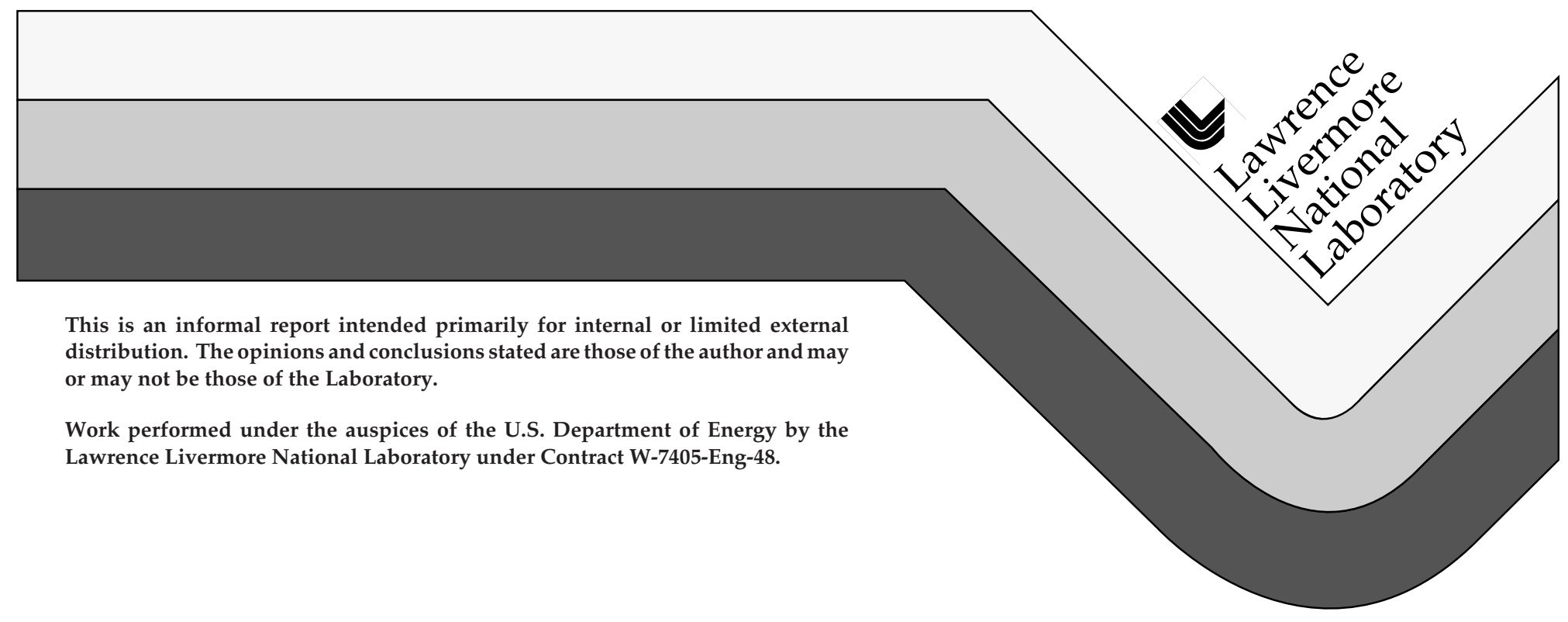




\section{DISCLAIMER}

This document was prepared as an account of work sponsored by an agency of the United States Government. Neither the United States Government nor the University of California nor any of their employees, makes any warranty, express or implied, or assumes any legal liability or responsibility for the accuracy, completeness, or usefulness of any information, apparatus, product, or process disclosed, or represents that its use would not infringe privately owned rights. Reference herein to any specific commercial product, process, or service by trade name, trademark, manufacturer, or otherwise, does not necessarily constitute or imply its endorsement, recommendation, or favoring by the United States Government or the University of California. The views and opinions of authors expressed herein do not necessarily state or reflect those of the United States Government or the University of California, and shall not be used for advertising or product endorsement purposes.

This report has been reproduced directly from the best available copy.

Available to DOE and DOE contractors from the Office of Scientific and Technical Information

P.O. Box 62, Oak Ridge, TN 37831

Prices available from (615) 576-8401, FTS 626-8401

Available to the public from the

National Technical Information Service

U.S. Department of Commerce 5285 Port Royal Rd.,

Springfield, VA 22161 


\title{
Efficient Computation of Volume of Hexahedral Cells
}

\author{
Jeffrey Grandy \\ Lawrence Livermore National Laboratory
}

\begin{abstract}
We describe an efficient method to compute the volume of hexahedral cells used in three-dimensional hydrodynamics simulations. We consider two common methods for treating the hexahedron using triangular boundaries.
\end{abstract}

\section{Motivation}

In Lagrangian and ALE hydrodynamics simulations, a crucial step in the physics calculation is to compute the volume of a zone, which is used to determine the density and thermodynamics properties of the material from the mass in the physical Lagrangian cycle, and also to find the volume of zones after remapping the mesh in ALE codes. Since the volume of every zone must be computed at least once per time step this represents a significant part of the problem computation time and it therefore behooves us to accomplish the volume calculation in as few as possible floating point operations (Flops). We focus on simulations using hexahedral zones, which are specified by the locations of eight nodes logically connected as a cube.

\section{Cell Definition}

The definition of the volume of the hexahedron (hex) depends on the method used to construct surfaces between the twelve edges of the logical cube. Since the nodes are allowed to move independently of each other in the physics simulation the four edges surrounding a face of the logical cube are not in general coplanar, and we construct planar boundaries for the hex cell by dividing the cube face into triangles. There are several methods for developing a set of triangles, and we consider two of these methods. One method is to use the long diagonal (LD) method for splitting the hex into six tetrahedra (tets), thus defining a twelve-faceted triangular polyhedron to represent the hex, a polyhedron that is isomorphic to a hexagonal dipyramid. This method introduces directional preferences along the diagonals selected for triangulation, a broken symmetry which is undesirable from a physics standpoint. Another method, preserving the diagonal symmetry, is to define an additional vertex at the barycenter of each face, and construct triangles containing the barycenter and each surrounding edge. These additional vertices are

$$
\begin{aligned}
\vec{x}_{e} & =\left(\vec{x}_{1}+\vec{x}_{3}+\vec{x}_{5}+\vec{x}_{7}\right) / 4 \\
\vec{x}_{w} & =\left(\vec{x}_{0}+\vec{x}_{2}+\vec{x}_{4}+\vec{x}_{6}\right) / 4 \\
\vec{x}_{n} & =\left(\vec{x}_{2}+\vec{x}_{3}+\vec{x}_{6}+\vec{x}_{7}\right) / 4 \\
\vec{x}_{s} & =\left(\vec{x}_{0}+\vec{x}_{1}+\vec{x}_{4}+\vec{x}_{5}\right) / 4 \\
\vec{x}_{t} & =\left(\vec{x}_{4}+\vec{x}_{5}+\vec{x}_{6}+\vec{x}_{7}\right) / 4 \\
\vec{x}_{b} & =\left(\vec{x}_{0}+\vec{x}_{1}+\vec{x}_{2}+\vec{x}_{3}\right) / 4
\end{aligned}
$$

Each face is divided into four triangles, and the hex is defined by a 24 -faceted triangular polyhedron known as a tetrakis hexahedron $(\mathrm{TH})$ (Weisstein, 1997). These two definitions of a hex are illustrated in Figure 1.

\section{Volume Calculation}

We now discuss the relative efficiency of volume algorithms for a $\mathrm{TH}$ zone, which is commonly used in physics simulations. We number the eight original nodes as in Figure 1. A straightforward method for computing the volume is

$$
6 v=\sum_{\substack{\left.1 \\\left(x_{r}-x_{p}\right)\left(y_{q}-y_{p}\right)\right)}}^{24}\left(z_{p}+z_{q}+z_{r}\right)\left(\left(x_{q}-x_{p}\right)\left(y_{r}-y_{p}\right)-\right.
$$

where the sum is over the 24 triangles and the vertices $p, q, r$ are oriented with the triangle normal pointing outward from a simple hex. By inspection this formula takes 264 Flops: 10 for each term, 23 to add the terms, and one overall multiplication. By reusing the differences in $x$ and $y$ coordinates we can reduce the operation count to an average of 8 per term, for a total of 216 Flops. This method is prone to numerical cancellations 


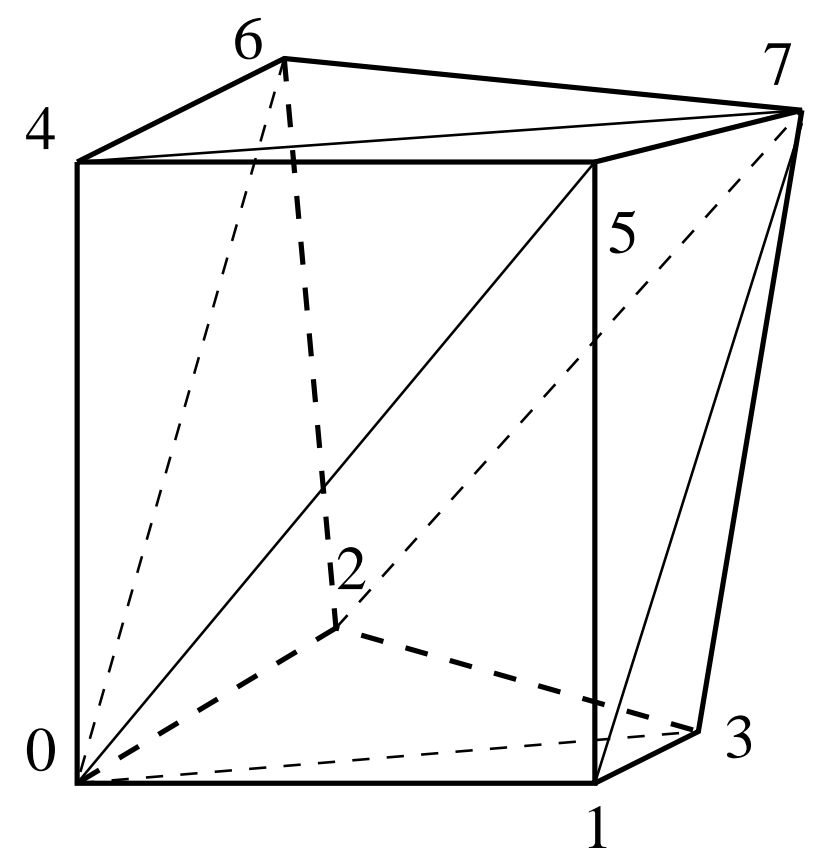

a)

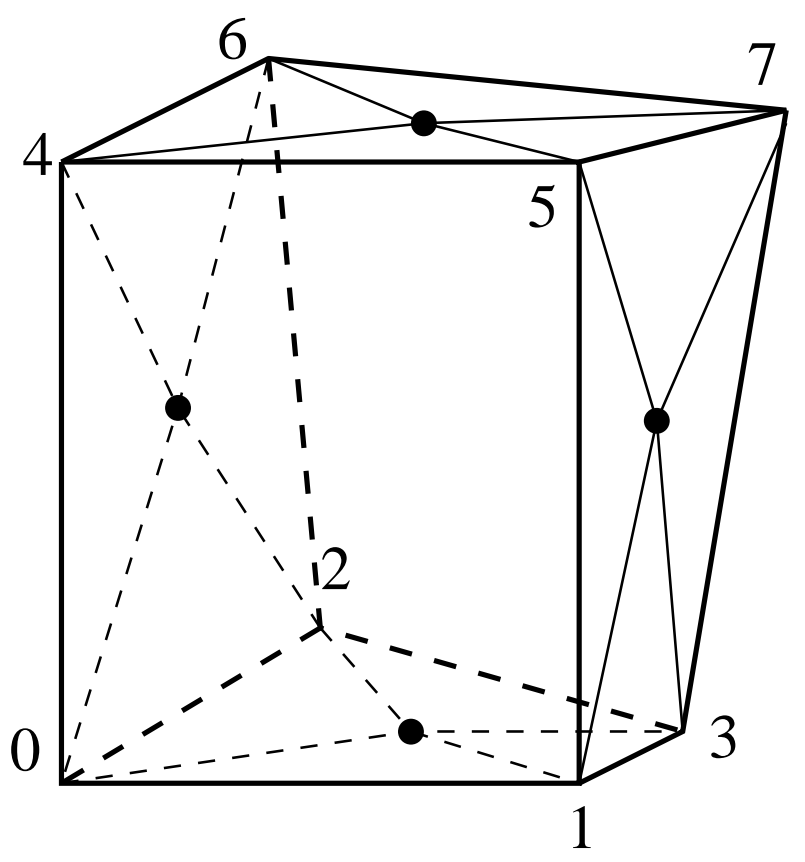

b)

Figure 1: Definitions of hexahedral cells from a)long diagonal triangulation, and b)tetrakis hexahedron with face-centered vertices (subdivision of front and back faces not drawn). Logical numbering of nodes is given.

because the $z$ coordinates are entered into multiplication without first subtracting an offset.

Another common algorithm is to select a central point of the TH (the barycenter $x_{c}$, or average of the eight nodes, is a convenient choice) and to construct tetragonal dipyramids (octahedra) containing the central point, the four nodes surrounding the face, and the vertex at the barycenter of the face (Figure 2). The volume of each octahedron is computed using the standard formula:

$$
\begin{aligned}
v_{\text {oct }}= & 1 / 12\left[\vec{x}_{c}-\vec{x}_{b}, \vec{x}_{0}-\vec{x}_{1}+\vec{x}_{2}-\vec{x}_{3},\right. \\
& \left.\vec{x}_{0}+\vec{x}_{1}-\vec{x}_{2}-\vec{x}_{3}\right]
\end{aligned}
$$

where $[\vec{A}, \vec{B}, \vec{C}]$ is the triple product ${ }^{a}$

$$
[\vec{A}, \vec{B}, \vec{C}]=\left|\begin{array}{lll}
A_{x} & B_{x} & C_{x} \\
A_{y} & B_{y} & C_{y} \\
A_{z} & B_{z} & C_{z}
\end{array}\right| .
$$

By reusing the edge differences, we can use this method to compute the volume of a TH in 199

${ }^{a}$ The author prefers the bracket notation rather than the commonly used $\vec{A} \cdot(\vec{B} \times \vec{C})$ since the latter does not explicitly suggest equal roles for the three vectors.
Flops. This algorithm uses the formula for a general octahedron and therefore does not use the fact that $x_{b}$ is known to be located at the average of the four face nodes.

We now develop a different method to find the volume of a $\mathrm{TH}$ zone, which does take advantage of this fact. We write the volume of the zone by constructing tets, one of whose vertices is $\vec{x}_{0}$. The volume associated with the logical face defined by nodes $1,3,7,5$, which is opposite to node 0 , is

$$
\begin{aligned}
6 v_{1375}= & {\left[\left(\vec{x}_{1}-\vec{x}_{0}\right),\left(\vec{x}_{3}-\vec{x}_{0}\right),\left(\vec{x}_{e}-\vec{x}_{0}\right)\right]+} \\
& {\left[\left(\vec{x}_{3}-\vec{x}_{0}\right),\left(\vec{x}_{7}-\vec{x}_{0}\right),\left(\vec{x}_{e}-\vec{x}_{0}\right)\right]+} \\
& {\left[\left(\vec{x}_{7}-\vec{x}_{0}\right),\left(\vec{x}_{5}-\vec{x}_{0}\right),\left(\vec{x}_{e}-\vec{x}_{0}\right)\right]+} \\
& {\left[\left(\vec{x}_{5}-\vec{x}_{0}\right),\left(\vec{x}_{1}-\vec{x}_{0}\right),\left(\vec{x}_{e}-\vec{x}_{0}\right)\right] }
\end{aligned}
$$

and substitute (1) into (5) to obtain

$$
\begin{aligned}
12 v_{1375}= & {\left[\left(\vec{x}_{1}-\vec{x}_{0}\right),\left(\vec{x}_{3}-\vec{x}_{0}\right),\left(\vec{x}_{5}-\vec{x}_{0}\right)\right]+} \\
& {\left[\left(\vec{x}_{5}-\vec{x}_{0}\right),\left(\vec{x}_{1}-\vec{x}_{0}\right),\left(\vec{x}_{7}-\vec{x}_{0}\right)\right]+} \\
& {\left[\left(\vec{x}_{1}-\vec{x}_{0}\right),\left(\vec{x}_{3}-\vec{x}_{0}\right),\left(\vec{x}_{7}-\vec{x}_{0}\right)\right]+} \\
& {\left[\left(\vec{x}_{3}-\vec{x}_{0}\right),\left(\vec{x}_{7}-\vec{x}_{0}\right),\left(\vec{x}_{5}-\vec{x}_{0}\right)\right](6) }
\end{aligned}
$$

For the face 0231 , which contains node 0 , the volume is simply

$$
12 v_{0231}=\left[\left(\vec{x}_{1}-\vec{x}_{0}\right),\left(\vec{x}_{2}-\vec{x}_{0}\right),\left(\vec{x}_{3}-\vec{x}_{0}\right)\right]
$$




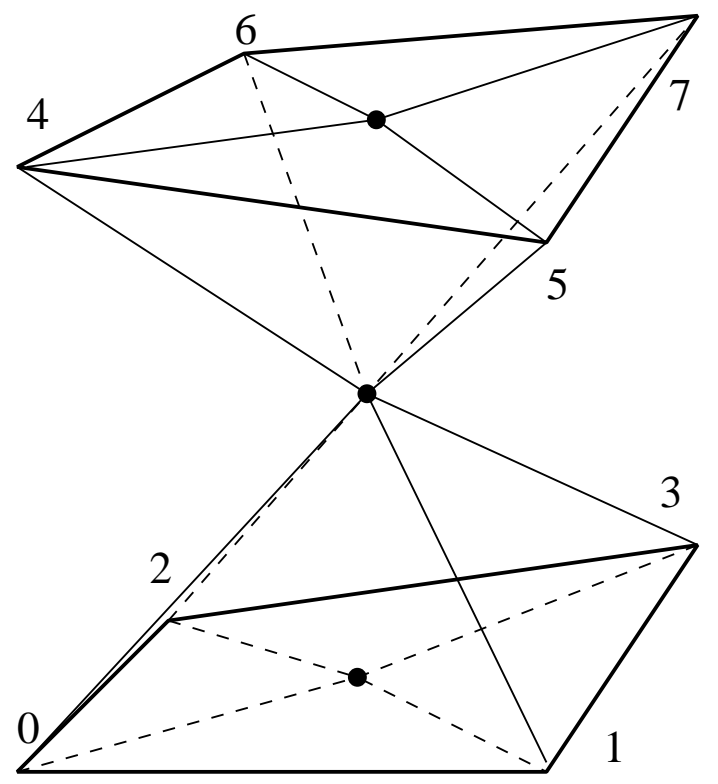

Figure 2: Two of the six octahedra in the decomposition of a 24 -sided hex cell.

and the volume for the entire $\mathrm{TH}, v_{T H}$, is obtained by adding (6), (7), and corresponding expressions for the other four faces:

$$
\begin{aligned}
v_{T H}= & v_{1375}+v_{4576}+v_{2673}+ \\
& v_{0231}+v_{0154}+v_{0462}
\end{aligned}
$$

We define the quantity $v_{1}$ as the Jacobian of the transformation from logical space to physical space at the point $x_{c}$,

$$
v_{1}=\left[\left(\vec{x}_{t}-\vec{x}_{b}\right),\left(\vec{x}_{e}-\vec{x}_{w}\right),\left(\vec{x}_{n}-\vec{x}_{s}\right)\right]
$$

which reduces to

$$
\begin{aligned}
16 v_{1}= & {\left[\left(\vec{x}_{7}-\vec{x}_{1}+\vec{x}_{6}-\vec{x}_{0}\right),\right.} \\
& \left(\vec{x}_{7}-\vec{x}_{2}+\vec{x}_{5}-\vec{x}_{0}\right), \\
& \left.\left(\vec{x}_{7}-\vec{x}_{4}+\vec{x}_{3}-\vec{x}_{0}\right)\right]
\end{aligned}
$$

We write the columns of (10) as

$$
\left(\vec{x}_{7}-\vec{x}_{0}\right)-\left(\vec{x}_{1}-\vec{x}_{0}\right)+\left(\vec{x}_{6}-\vec{x}_{0}\right)
$$

etc., expand (10), and compare with (8), to obtain

$$
\begin{aligned}
12 v_{T H}= & 16 v_{1}- \\
& \left(\left[\left(\vec{x}_{6}-\vec{x}_{0}\right),\left(\vec{x}_{5}-\vec{x}_{0}\right),\left(\vec{x}_{3}-\vec{x}_{0}\right)\right]+\right. \\
& {\left.\left[\left(\vec{x}_{7}-\vec{x}_{1}\right),\left(\vec{x}_{7}-\vec{x}_{2}\right),\left(\vec{x}_{7}-\vec{x}_{4}\right)\right]\right) }
\end{aligned}
$$

where the triple products in (11) are positive for a unit cube, and therefore this formula subjects the volume to a slight subtractive cancellation. This subtraction is easily removed by substituting (10)

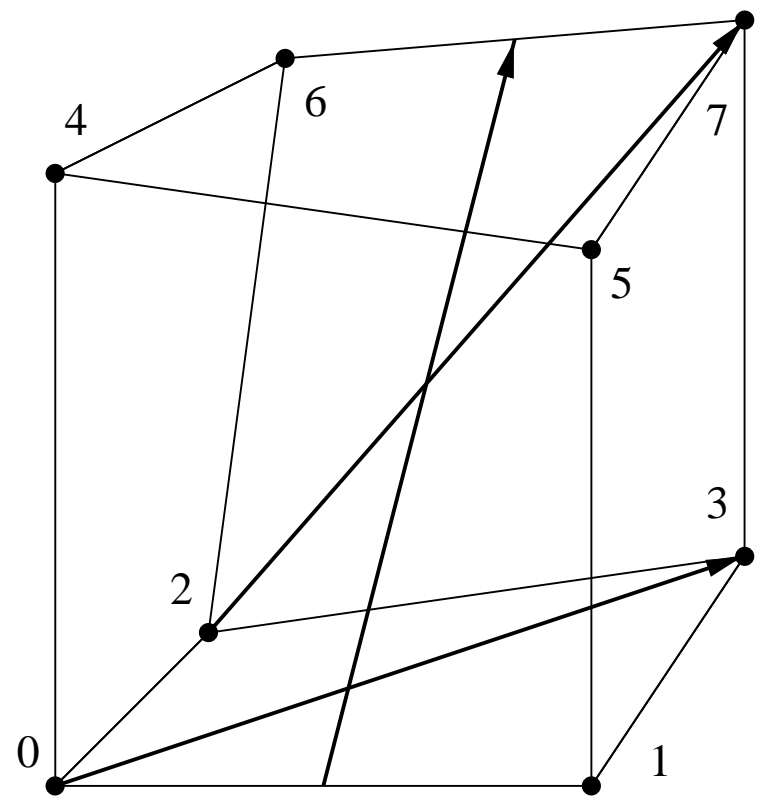

Figure 3: Graphical representation of the first term in (12). The three vectors in the triple product are the logical-plane diagonals 03 and 27, and the average of 06 and 17. The other two terms are cyclic permutations of these logical-plane diagonals.

into (11), and the result is

$$
\begin{aligned}
12 v_{T H}= & {\left[\left(\vec{x}_{7}-\vec{x}_{1}\right)+\left(\vec{x}_{6}-\vec{x}_{0}\right)\right.} \\
& \left.\left(\vec{x}_{7}-\vec{x}_{2}\right),\left(\vec{x}_{3}-\vec{x}_{0}\right)\right]+ \\
& {\left[\left(\vec{x}_{6}-\vec{x}_{0}\right),\left(\vec{x}_{7}-\vec{x}_{2}\right)+\left(\vec{x}_{5}-\vec{x}_{0}\right)\right.} \\
& \left.\left(\vec{x}_{7}-\vec{x}_{4}\right)\right]+ \\
& {\left[\left(\vec{x}_{7}-\vec{x}_{1}\right),\left(\vec{x}_{5}-\vec{x}_{0}\right)\right.} \\
& \left.\left(\vec{x}_{7}-\vec{x}_{4}\right)+\left(\vec{x}_{3}-\vec{x}_{0}\right)\right] .
\end{aligned}
$$

All of the differences in parentheses in (12) are plane diagonals, and one of the terms is illustrated in Figure 3. This algorithm for computing $v_{T H}$ requires 27 Flops to find sums and differences of node coordinates, three $(3 \times 3)$ determinants with 14 Flops each, and three additional Flops for adding and normalizing the volume, for a total of 72 Flops to compute $v_{T H}$. The formula (12) has been applied in a mesh generation code.

\section{Volume of LD Hexahedron}

The LD hexahedron (Figure 1a) comprises 12 triangular facets and eight vertices whose coordinates are in general unrelated to each other. A generic method for computing the volume is to treat this LD hex as a hexagonal dipyramid, decomposing it into two octahedra and using the formula for an octahedron volume described above. 


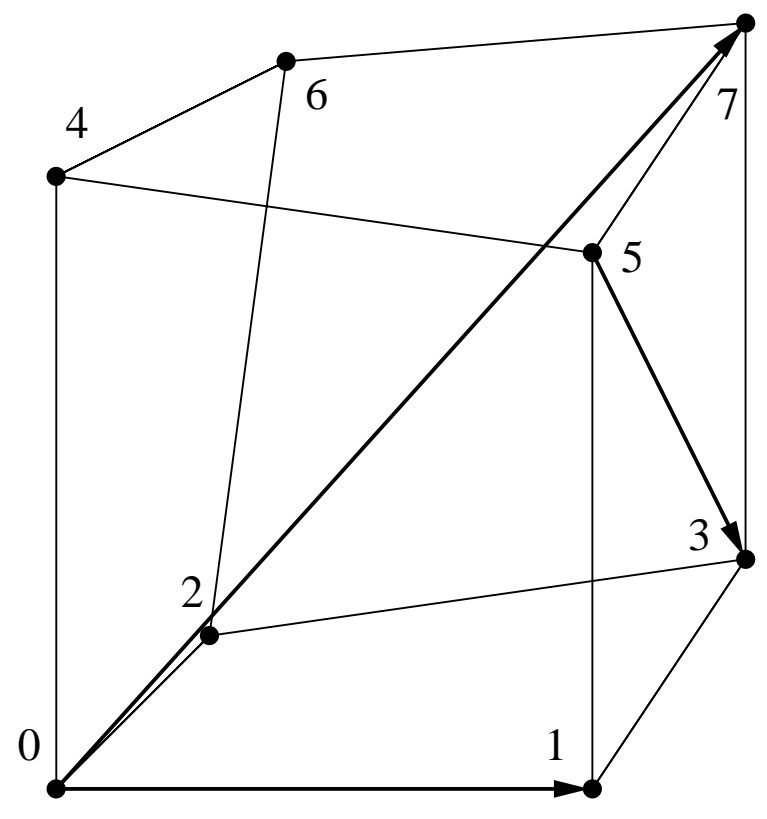

Figure 4: Graphical representation of the first term in (14). The triple product contains the body diagonal connecting the two apex nodes 0 and 7 , a logical plane diagonal opposite to the triangulation, and an edge. The other two terms are cyclic permutations of the edge and plane vectors.

The following expression for the volume $v_{L D}$ represents one such decomposition:

$$
\begin{aligned}
12 v_{L D}= & {\left[\left(\vec{x}_{7}-\vec{x}_{0}\right),\right.} \\
& \left(\vec{x}_{1}+\vec{x}_{6}\right)-\left(\vec{x}_{4}+\vec{x}_{5}\right), \\
& \left.-\left(\vec{x}_{1}-\vec{x}_{6}\right)+\left(\vec{x}_{4}-\vec{x}_{5}\right)\right]+ \\
& {\left[\left(\vec{x}_{7}-\vec{x}_{0}\right),\right.} \\
& \left(\vec{x}_{1}+\vec{x}_{6}\right)-\left(\vec{x}_{3}+\vec{x}_{2}\right), \\
& \left.\left(\vec{x}_{1}-\vec{x}_{6}\right)+\left(\vec{x}_{3}-\vec{x}_{2}\right)\right]
\end{aligned}
$$

Since nodes 0 and 7 are the apex points of the LD triangulation, they enter $v_{L D}$ differently from the other six nodes. In equation (13) nodes 1 and 6 are the joints of the decomposition into octahedra; one could have selected 2 and 5, or 3 and 4 . This expression requires 33 Flops to precompute the sums and differences of vectors, 28 Flops for two determinants, and two flops at the end to combine and normalize, for a total of 63 Flops.

Alternatively one may compute $v_{L D}$ using three $(3 \times 3)$ determinants with simpler constituents:

$$
\begin{aligned}
6 v_{L D}= & {\left[\left(\vec{x}_{7}-\vec{x}_{0}\right),\left(\vec{x}_{1}-\vec{x}_{0}\right),\left(\vec{x}_{3}-\vec{x}_{5}\right)\right]+} \\
& {\left[\left(\vec{x}_{7}-\vec{x}_{0}\right),\left(\vec{x}_{4}-\vec{x}_{0}\right),\left(\vec{x}_{5}-\vec{x}_{6}\right)\right]+} \\
& {\left[\left(\vec{x}_{7}-\vec{x}_{0}\right),\left(\vec{x}_{2}-\vec{x}_{0}\right),\left(\vec{x}_{6}-\vec{x}_{3}\right)\right] }
\end{aligned}
$$

Algorithm (14) requires seven vector subtractions (21 Flops), and three determinants. However, since the first column is the same for all three terms, we can combine the $(2 \times 2)$ minors and compute the sum of determinants in 38 Flops. Including the overall normalization brings the total for (14) to 60 Flops. We therefore obtain an advantage by using three determinants since less precomputations of vector sums and differences are needed than for (13). An illustration of the first term of (14) is shown in Figure 4.

\section{Summary}

We have studied algorithms for computing two different commonly used definitions of the volume of hexahedral cells, such as are used in hydrodynamics simulations. For the face-centered (24-faceted) type of hexahedron, we have shown that the volume can be computed in 72 Flops. For the 12-faceted hexahedron derived from a long diagonal decomposition, the volume can be computed with 60 Flops. We plan to present a detailed comparison of roundoff properties of the various volume algorithms in a followup paper.

\section{Acknowledgments}

This work was performed under the auspices of the United States Department of Energy by Lawrence Livermore National Laboratory under contract number W-7405-Eng-48. The author would like to thank Gary Carlson, Tom McAbee, Chris Clouse, and Robert Tipton for helpful discussions.

\section{References}

Weisstein, E., Eric's Treasure Troves of Science, http://www.astro.virginia.edu/ eww6n/math/ (1997). 


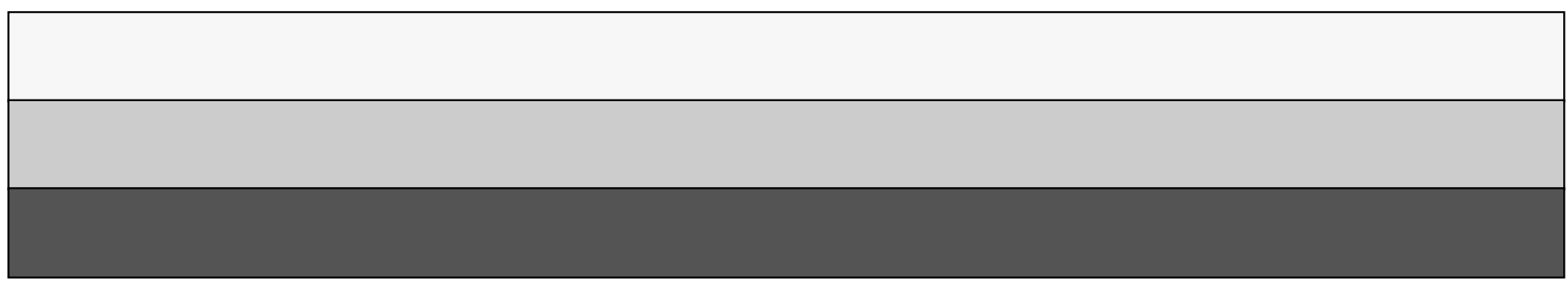

